\title{
BUILDING A REUSABLE DATA MODEL TO SUPPORT SYSTEMATIC LAY- OUT PLANNING WITH DISCRETE EVENT SIMULATION OF FLEXIBLE MANUFACTURING SYSTEMS
}

\author{
ZSOlt MOLNÁR*1, PÉTER TAMÁS ${ }^{1}$, AND BÉLA ILLÉS ${ }^{1}$ \\ ${ }^{1}$ Institute of Logistics, University of Miskolc, Egyetemváros, Miskolc, 3515, HUNGARY
}

Manufacturing has undergone a greater change in recent years than it has over almost the entire past century. Due to the variety of products, shortening delivery times and changing customer habits, the COVID-19 pandemic has demanded greater flexibility from manufacturing companies than ever before. One of the fundamental parts of manufacturing flexibility is the design of manufacturing layouts, which has so far not followed automation and methodological developments with regard to technological areas of manufacturing. In this article, a new method for supporting traditional factory design methods with digital manufacturing tools is introduced.

Keywords: flexible manufacturing, simulation, layout design, Systematic Layout Planning

\section{Introduction}

Very few complete methodologies cover and support the entire process of designing manufacturing layouts. When the designing of a layout is considered, what are the most important parameters? The space available and the complexity of the manufacturing process of the product, which is directly proportional to the material flow and the costs, immediately come to mind. Our goal was to design an extendable method to be supported by digital manufacturing tools for the purposes of improving as well as speeding up the design and evaluation of future manufacturing layouts.

In terms of project planning, the study by Schenk et al. [1] comprehensively covers the design of factories and production lines but deals relatively little with the specific challenges of implementation. Their $0+5+X$ method is much more of a project planning and management method.

The study by Wiendahlet al. [2] presents many more concrete design methods. In addition to examining workplace design, including the connection between layout elements, it handles the workplace, work area, building and site design separately, albeit only to a limited extent, for the purpose of integrating these aspects into a complete system.

To the best of our knowledge, the Systematic Layout Planning (SLP) methodology by Muther and Hales [3] is the most comprehensive methodology to date. It addresses the data requirements of layout design and creates

*Correspondence: zsolt.molnar.zsolt@outlook.com production layouts by following a top-down approach. The design methodology takes into consideration relationships, space and adjustments. As this methodology also contains practical examples, templates and formulae for the steps of layout designs, this method is best suited for use with digital tools. In its current state, this method mainly focuses on traditional manufacturing processes and does not include specific methods for the design of Flexible Manufacturing Systems (FMS) [4] or Reconfigurable Manufacturing Systems (RMS).

Since this method was developed in the 1970s, it was mainly designed for paper-based workflows used to design classic dedicated manufacturing lines. Given the advancement of FMSs, understanding to what extent SLP can be supported by digital manufacturing as well as simulation tools and thus to what degree it can be used to design FMS's is a current research topic.

During the research, the Siemens Plant Simulation was used as a platform for the digital twin. The Siemens Plant Simulation is a discrete event-based piece of simulation software that takes into account a wide range of factory objects, e.g. material flow and logistics, as well as customization possibilities via its built-in programming environment [5,6]. Another advantage of the system is that since the table component it contains is very versatile, it is also suitable for handling tabular and matrix data [7].

\section{Digital twin}

The digital twin has now become a basic tool for the development of production processes. A digital twin is 
Table 1: Products and bill of materials

\begin{tabular}{cccc}
\hline Product & Component & Qty & Operation number \\
\hline A & B & 3 & 20 \\
C & B & 2 & 10 \\
A & D & 1 & 10 \\
\hline
\end{tabular}

Table 2: A typical product routing

\begin{tabular}{ccc}
\hline Product & Operation number & Activity Point \\
\hline $\mathrm{A}$ & 10 & M1 or M2 \\
& 20 & M3 \\
\hline
\end{tabular}

the mapping of production in a digital model to develop the production process within the framework of the digital model. The depth of formation of the digital twin is determined in each case by the development goal [8]. Process development in the digital model is typically cheaper, providing more what-if analysis possibilities than an analysis or trial of a real-life production system [9].

During the current research, the first steps to digitalize the SLP method and make it available to use for the design of flexible manufacturing lines were taken.

\section{Data model}

SLP works according to 5 elements: product, quantity, routing, supporting service and timing. The implications of these 5 groups of data on flexible manufacturing systems is outlined below.

In the case of products, the main purpose of flexible manufacturing systems is to facilitate the production of several products as a result of their flexibility. FMS's always try to implement high-mix and low volume production. The product data should include the related bill of materials along with information outlining which operation refers to the related necessary quantity of a part that is assembled or used to build the product. Example product data with information concerning the bill of materials is shown in Table 1

In terms of FMS lines, the most important parameter is routing, which determines the flexibility of the line itself. A typical product route with the order of operations and the machines (activity points) that are capable of executing the process step is shown in Table 2 . An activity point is a location where something happens to a product. Details of this definition will be provided and expanded on later. Although the order of operations is usually fixed (or hardly modifiable), most of these lines include a machine to substitute bottleneck resources to make routing more flexible.

The routing data should include the product data and process steps, including the alternative routings which are very common in flexible manufacturing cells.

A typical product routing is presented below.

In the case of flexible manufacturing, the data model should be able to handle the alternative routings. For the
Table 3: First alternative routing of Product A

\begin{tabular}{ccc}
\hline Product & Operation number & Activity Point \\
\hline $\mathrm{A}$ & 10 & M1 or M2 \\
& 20 & M3 \\
\hline
\end{tabular}

Table 4: Second alternative routing of Product A

\begin{tabular}{ccc}
\hline Product & Operation number & Activity Point \\
\hline A & 10 & M3 \\
& 20 & M1 or M2
\end{tabular}

sake of clarity, each alternative routing was stored separately along with the full routing data, thereby ensuring that it can be handled faster and more easily with a route search algorithm. Tables 3 and 4 show alternative routings for the same product.

Given the routing tables, the question may arise as to where to store product-dependent parameters during the simulation, e.g., how long should the production time of Product A be on machines M1 and M2? The data model follows the Industry 4.0 philosophy of storing data where data-related decisions are made, that is, on machines. In this case, machine M1 knows how much time Product A spends on it and this information can be made available to other elements of the production system at any time.

The most important areas of the supporting services are maintenance and raw material supply. Both are critical to the operation of the line. Although the supply of raw materials influences the design and layout at a basic level, maintenance is vital to ensure a functional operation. Since operational services are mostly machinerelated, they must be handled by the machine (activity point).

Repair and rework are special supporting services. If quality control and repair take place within the machine, the necessary time can be regarded as the duration of a process step. If quality control is carried out elsewhere, it should be treated and mapped as part of the routing.

The time-based parameter of the SLP design process can determine when items will be produced. Usually, the number of pieces and product mix over a given period, e.g. monthly, are available here. Based on this, orders can be generated in the simulation, the distribution of which corresponds to the given data.

The relationship between the SLP layout design process and the simulation is shown in Fig. 1. Apparently, the steps of the design process can be mapped to the steps of building a simulation model.

\section{Activity points}

During a manufacturing process, a part could be subjected to six activities:

1. Can be processed (assembled, disassembled or manufactured in any way)

2. Can be moved or transported 


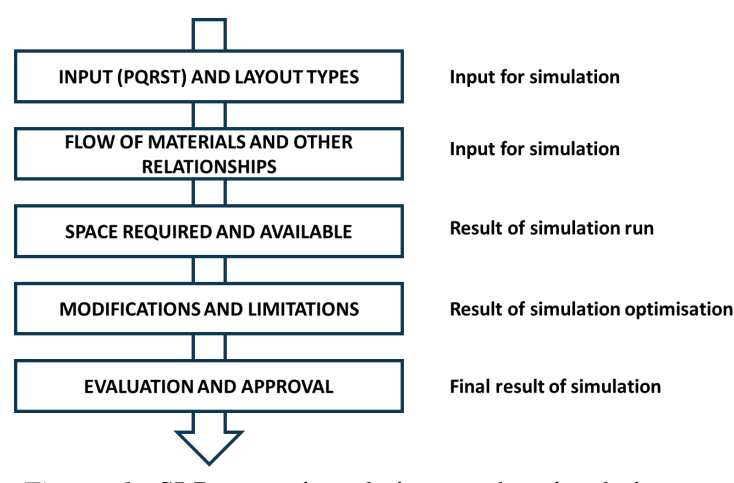

Figure 1: SLP steps in relation to the simulation process [1]

3. Can be handled to support processing (change in orientation, placed in a tool, etc.)

4. Can be handled to enhance quality (inspected, tested, counted, repaired or reworked)

5. Can be forced to wait due to technological reasons (to cool down, etc.) or for the rest of the batch to catch up

6. Can be stored

To manage activities uniformly, the concept of an activity point was introduced, which is a generic object that can handle the aforementioned six activities in one object depending on the settings specified.

A manufacturing object, e.g., an activity point, can be a milling machine, 3D printer or even a buffer as shown in Fig. 2.

The main attributes of an activity point are:

- Object type (processing, handling, transferring, buffering, quality handling, storage)

- Processing type

- Processing time (product-dependent)

- Setup parameters

- Capacity

- 3D block size parameters (x, y, z)

- Logic of supporting services

With the help of activity points, the simulation model can be easily built and modified.

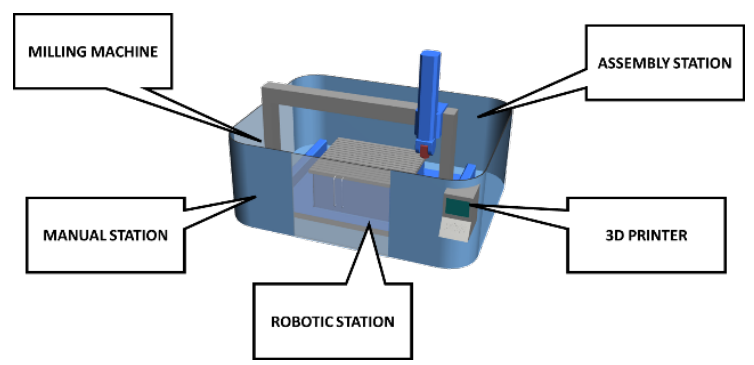

Figure 2: An activity point can represent different types of process objects

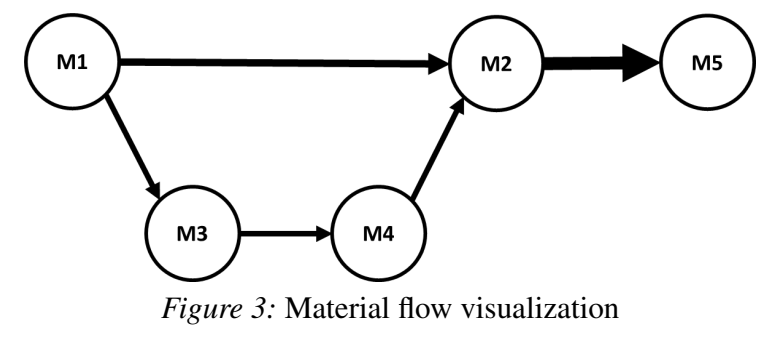

Depending on how comprehensive the analysis is, an activity point could be planned by a department or a single processing machine.

\section{Simulation results}

The simulation model is suitable for generating the results required for the further steps of the SLP analysis.

The simulation automatically creates a Sankey (or spaghetti) diagram characterizing the material flow intensity between each activity point (Fig. 3).

The most important result is the so-called Travel Chart that shows how much material has moved between each activity point. The upper diagonal of the table shows the amount of material that has moved forwards, while the quantity that has moved backwards, e.g. a loop in progress or returned for repair, is depicted below the diagonal. On the diagonal, all values are, by definition, zero.

Based on the data table, the material movement (or flow) between two activity points can be calculated, which is defined as:

$$
m_{i j}=\text { material flow from activity } i \text { to activity } j
$$

Due to the possible backward material flow, the total material movement between two activity points can be calculated by this formula:

$$
M_{i j}=m_{i j}+m_{j i}, \text { for all } i>j
$$

SLP uses an REL (relationship) chart to visualize the importance of how close the different departments are to each other. The REL chart replaces the numbers in the Travel Chart with a closeness category. Six categories are defined to represent the level of importance [3]:

- A - Absolutely necessary

- E - Very important

- I - Important

- O - Fairly important

- U - Unimportant

- X - Undesirable

Table 5: Travel Chart (From-To chart)

\begin{tabular}{cccc}
\hline & M1 & M2 & M3 \\
\hline M1 & 0 & $m_{12}$ & $m_{13}$ \\
M2 & $m_{21}$ & 0 & $m_{21}$ \\
M3 & $m_{31}$ & $m_{32}$ & 0 \\
\hline
\end{tabular}




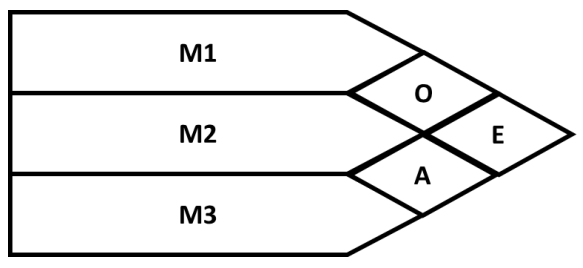

Figure 4: Simple chart depicting the importance of how close each activity is to each other

Table 6: Summarized Travel Chart

\begin{tabular}{cccc}
\hline & M1 & M2 & M3 \\
\hline M1 & 0 & $M_{12}$ & $M_{13}$ \\
M2 & - & 0 & $M_{21}$ \\
M3 & - & - & 0 \\
\hline
\end{tabular}

Table 7: Chart depicting the importance of the proximity of the activity points to each other

\begin{tabular}{cccc}
\hline & M1 & M2 & M3 \\
\hline M1 & 0 & O & E \\
M2 & - & 0 & A \\
M3 & - & - & 0 \\
\hline
\end{tabular}

In the example (Fig. 4), 3 departments (activity points) are present to evaluate our layout. The intersections denoted by letters depict the importance of how close the departments are to each other.

From the simulation model, the results of the Travel Chart can be transformed into a full Travel Chart (Table 6) which includes the total material flow between the activity points.

By taking the largest and smallest values from the table as well as dividing the range into 6 parts, six categories can be created to determine the importance of the proximity of the activity points to each other. The content of the resulting table is identical to the chart depicting how important the proximity between activity points is which is required to evaluate the SLP methodology (Table 7).

Based on the table, decisions can be made as to which departments and machines should be placed close to each other as well as how the layout elements should be designed.

\section{Summary and further research}

Supporting and further developing traditional factory design methods with digital manufacturing and simulation tools is a new approach to design better production lines and shorten design processes.

As the aforementioned method is rather generic, that is, applicable to many scenarios, it does not completely fulfill the special requirements of the production lines in FMS's or RMS's. The next phase of this research will strive to include the product mix, namely the variety of processes, in the model. Another important field of this research is how to evaluate an automation level of the production lines and determine which automation level is optimal by taking into consideration the material flow as well as technical and investment parameters.

\section{REFERENCES}

[1] Schenk, M.; Wirth, S.; Müller, E.: Factory Planning Manual, Springer-Verlag Berlin Heidelberg, 2010 ISBN: 978-3-642-03634-7, DOI: 10.1007/978-3-642-03635-4

[2] Wiendahl, H. P.; Reichardt, J.; Nyhuis, P.: Handbook of Factory Planning and Design, Springer Heidelberg New York Dordrecht London, 2015 ISBN: 978-3-662-46390-1, DOI: 10.1007/978-3-662-46391-8

[3] Muther, R.; Hales, L.: Systematic Layout Planning, Marietta, GA: Management \& Industrial Research Publications, 2015, ISBN 978-0-933684-06-5

[4] Koštál, P.; Velíšek, K.: Flexible Manufacturing System, Zenodo, 2011 DOI: 10.5281/zenodo.1075605

[5] Erdélyi, F.; Tóth, T.; Kulcsár, G. Y.; Mileff, P.; Hornyák, O.; Nehéz, K.; Körei, A.: New Models and Methods for Increasing the Efficiency of Customized Mass Production, J. Mach. Manuf., 2009, 49, 11-17

[6] Tamás, P.; Illés, B.; Tollár, S.: Simulation of a flexible manufacturing system, Adv. Logist. Syst. 2012, 6(1), 25-32

[7] Bratley, P.; Fox, B. L.; Schrage, L. E.: A Guide to Simulation, 2nd ed., Springer: New York, NY, USA, 1987 ISBN: 978-1-4612-6457-6.

[8] Cselényi, J.; Illés, B.: Planning and Controlling of Material Flow Systems (Textbook), Miskolc, Hungary: Miskolci Egyetemi Kiadó, 2006

[9] Dörgő, G.; Abonyi, J.: Group Contribution Methodbased Multi-objective Evolutionary Molecular Design. Hung. J. Ind. Chem., 2016, 44(1), 39-49 DOI: 10.1515/hjic-2016-00056 